\title{
50-GHz Repetition Gain Switching Using A Cavity-enhanced DFB Laser Assisted By Optical Injection Locking
}

\author{
Zhixin Liu, Yasuhiro Matsui, Richard Schatz, Ferdous Khan, Martin Kwakernaak, Tsurugi Sudo
}

\begin{abstract}
We demonstrate pulse generation at a repetition rate of $50 \mathrm{GHz}$ by gain switching an injection-locked distributed feedback (DFB) laser with large modulation bandwidth (BW). The small-signal BW of the DFB laser was extended from an intrinsic BW of $30 \mathrm{GHz}$ to $52 \mathrm{GHz}$ due to the joint effects of photon-photon resonance (PPR) and detuned-loading, which exploits the frequency-dependent cavity loss to enhance the effective differential gain and thereby the relaxation oscillation frequency. The results show that PPR and detuned-loading effects can also be utilized to improve large-signal gain-switching, despite the dynamic changes of the detuned loading condition due to the large signal chirp.

By modulating the laser with 50-GHz RF signals, we obtained 50-GHz repetition rate pulses from the gain-switched laser, confirming that the BW enhancement effects are still valid for large-signal modulation. After that, we optically injection lock the gain-switched laser with strong external seeding light, which suppresses the chirp and creates frequency tones with $50 \mathrm{GHz}$ spacing. The optical injection locking (OIL) further improves the large-signal BW, resulting in narrower pulse width.

The spectrum of the gain-switched OIL directly modulated laser (OIL-DML) showed three sideband peaks above $70 \%$ from the peak (full width half maximum of $130 \mathrm{GHz}$ ), and a pulse width of $9.5 \mathrm{ps}$ (6.2 ps after deconvolution) was obtained. The results indicate that the pulse could be compressed to $2.4 \mathrm{ps}$, assuming linear chirp.
\end{abstract}

Index Terms-Directly Modulated Lasers, Optical Injection Locking, Photon-Photon Resonance, Gain Switching

\section{INTRODUCTION}

$\mathrm{D}$ IRECTLY modulated lasers (DML) have been used in optical communications ever since the birth of semiconductor lasers. The features of low cost and low power consumption make DMLs promising in cost and size sensitive markets such as data center interconnections, high performance computing and access networks [1].

In data communications, the DMLs are typically biased 3-5 times higher than the threshold current and the typical extinction ratio is around $4 \mathrm{~dB}$. The laser is modulated without

This Manuscript received xxx, 2018; revised xxx; accepted xxx. Date of publication xxx; date of current version xxx. This work was supported by the UK Engineering and Physical Sciences Research Council under EP/R041792/1, EP/R035342/1, and Royal Society RSGIR1\180200.

Z. Liu is with the Department of Electronics and Electrical Engineer at University College London, London, WC1E 7JE, U.K. (e-mail: zhixin.liu@ucl.ac.uk;). completely turning off the laser (i.e. the laser current is always above the threshold), to avoid distortion of the directly modulated signal caused by the overshooting or undershooting in the bit transitions [2]. Such overshooting dynamics, however, can be exploited for pulse generation by biasing the DML below threshold current and modulating with large driving signals, known as gain-switching technique [3]. The sudden increase of driving current allows the carriers to build up to a few times higher than the threshold carrier density before photon density starts to increase. The overshooting of the photons quickly depletes the stored carriers, terminating the stimulated emission of photons. In this gain-switching process, an optical pulse is generated with a nearly linear frequencydown chirp of $>100 \mathrm{GHz}$ across the pulse. This chirp, known as transient chirp [4], is typically 5-10 times higher than the adiabatic chirp that dominates in small-signal direct laser modulation. This interplay between the carriers and photons causes a relaxation oscillation which determines the pulse width delivered from the laser cavity. Due to the strong and linear down-frequency chirp the optical spectrum broadens and the generated pulse can be compressed using negative dispersion. For example, using a gain-switched laser followed by linear chirp compression, 12-ps-width pulses were compressed to 4ps-width pulses at a repetition rate of $20 \mathrm{GHz}$ [5]. The broad spectrum and short pulses result in high order modulation sidebands that create a frequency comb. The gain-switching of semiconductor laser diodes provides a compact and low cost method to generate periodic short pulses and frequency combs, finding applications in high baud rate data transmission, clock synchronization and optical signal processing [6-8]. Compared to mode-locked lasers, gain-switched lasers offer the flexibility to adjust the pulse repetition rate by changing the frequency of the RF driving signal.

Nevertheless, the relatively large timing jitter, relative intensity noise (RIN), and the incoherence of the consecutive pulses limit the application of gain-switched lasers in modern coherent communication systems. The issue stems from the fact that the gain-switched pulses build up each time from the

Y. Matsui, F. Khan, M. Kwakernaak, and T. Sudo are with II-VI Incorporated, Fremont, CA 94538 USA (e-mail: yasuhiro.matsui@ finisar.com; ferdous.khan@finisar.com; Tsurugi.Sudo@finisar.com).

R. Schatz is with the School of Information and Communication Technology, Royal Institute of Technology, Kista SE-164 40, Sweden (e-mail: rschatz@kth.se). 
spontaneous emission below the threshold, therefore there is no correlation between the pulses. This problem can be overcome with optical injection locking (OIL) [9]. By using OIL, the pulses build up from the master light, and therefore, the train of pulses becomes coherent and the timing jitter can be suppressed. This significantly improves the performance of the gain-switched lasers, demonstrating coherent pulses (or frequency comb) generation with reduced chirp, jitter and linewidth $[10,11]$. However, the repetition rate (or the frequency) of pulses is limited to about $30 \mathrm{GHz}$ due to the limited relaxation oscillation frequency, defined as $f_{r}$, of the gain-switched lasers [12]. This has constrained the applications of the gain-switched lasers in high baud rate coherent communications, which demand a free spectral range of comb sources to be $50 \mathrm{GHz}$ and above $[13,14]$.

It has been reported that $f_{r}$ needs to be close to the repetition frequency of gain switching in order to guarantee a singleperiod state of the pulse generation [15]. Thus, the $f_{r}$ must be close to $50 \mathrm{GHz}$ for $50-\mathrm{GHz}-$ repetition-rate pulse generation by gain switching. When the $f_{r}$ is lower than the repetition rate, the gain-switched lasers exhibit non-linear behavior including period-doubling state, Hopf bifurcation or the intermittency route to chaos [15]. It is also shown that injection seeding can control such non-linear behavior and suppress the occurrence of chaos [16].

To overcome the $f_{r}$ limit, a couple of approaches have been proposed to extend the DML BW. In [17], the passive feedback laser (PFL) approach was exploited to create a photon-photon resonance (PPR) between the external mode and the intrinsic distributed feedback (DFB) mode, resulting in a PPR frequency that enhances the small-signal modulation $\mathrm{BW}$ to $35 \mathrm{GHz}$ despite that the intrinsic $f_{r}$ of the PFL was only $12 \mathrm{GHz}$. The PPR effect exploits the presence of a side mode created by integrating a passive waveguide with a high reflection mirror at the end. Such side mode is well suppressed under CW bias condition. However, when the laser is modulated, the modulation sideband is injected into the side mode and resonantly amplified, which enhances the modulation response at the corresponding frequencies. However, since PPR mainly influences the small-signal BW but not the intrinsic carrierphoton resonance frequencies, its potential use for increasing the repetition rate for large-signal gain-switching has not been explored.

Another laser dynamic effect that can improve $f_{r}$ is detuned loading. Through a distributed Bragg reflector (DBR), modulation chirp is converted to the dynamic reduction of the cavity loss, which effectively increases the differential gain, and consequently, increases the $f_{r}$ [18]. The recently demonstrated distributed reflector (DR) laser exploits such effect and shows an $f_{r}$ of $30 \mathrm{GHz}$ [19]. Concurrently, the external cavity in the DR laser creates a PPR at around $50 \mathrm{GHz}$, resulting in a small-signal $\mathrm{BW}$ of $55 \mathrm{GHz}$ due to the joint effects of detuned loading and PPR [19]. Using 80-GBaud PAM-8 modulation, a data rate of $200 \mathrm{~Gb} / \mathrm{s}$ was demonstrated [20].

Yet another approach to increase $f_{r}$ is to harness the strong light confinement, $\Gamma$, by using membrane lasers [21]. With a high-thermal-conductivity substrate, an $f_{r}$ of about $40 \mathrm{GHz}$ was demonstrated [21]. The membrane laser is integrated with a passive waveguide with a $30 \%$ reflection to generate PPR effect at $95 \mathrm{GHz}$, achieving a modulation $\mathrm{BW}$ of $107 \mathrm{GHz}$ that supports $239 \mathrm{~Gb} / \mathrm{s}$ data transmission. However, detunedloading effect was not reported in this work.

Typically, PPR and detuned loading effects are sensitive to the position (wavelength) of the main lasing mode. Although these effects have been investigated in data communications with limited extinction ratio (e.g. the modulation current does not fall below threshold current), none of the aforementioned high-speed DMLs (PFL, DR laser, membrane laser) has been investigated for gain-switching. Therefore, the laser dynamics of these BW enhancement effects under extremely large chirped operation is not well understood.

In this paper, we report 50-GHz-repetition-rate pulse generation by gain-switching a cavity-enhanced DFB laser injection-locked by an narrow linewidth tunable laser. The cavity-enhanced DFB laser integrates a passive waveguide similar to PFL laser. However, a very weak reflection of $3 \%$ was applied at the end of the waveguide facet, instead of HR coating for PFL laser. This creates the detuned-loading effect to achieve an $f_{r}$ of $42 \mathrm{GHz}$ and simultaneously a PPR effect at 52 $\mathrm{GHz}$, realizing a corresponding $3-\mathrm{dB} \mathrm{BW}$ of $52.5 \mathrm{GHz}$. Different from the DR laser, which requires two current sources for the DFB and the DBR sections, respectively, the cavityenhanced DFB used in this experiment only has one contact for current driving, making it simpler and more cost-effective. By applying the OIL, an additional PPR was created at about $80 \mathrm{GHz}$ away from the main lasing mode.

By driving the injection-locked cavity-enhanced laser with $100 \mathrm{~mA}$ peak-to-peak modulation current, we generated optical pulses at a record-high repetition frequency of $50 \mathrm{GHz}$ with a side-mode suppression ratio (SMSR) of $>35 \mathrm{~dB}$. The observed pulse width was $6.2 \mathrm{ps}$ and $7.8 \mathrm{ps}$ (assuming $\mathrm{sech}^{2}$ pulses) with and without OIL, respectively. To the best of our knowledge, this represents the highest gain-switching speed and the shortest uncompressed pulse width (generated directly from gainswitched lasers) to date.

\section{LASER DYNAMICS}

The laser dynamics effects (detuned-loading, cavity PPR, and OIL) exploited in this work have been investigated individually for high-speed laser modulation and gain-switching. Here, we explain their concepts separately before discussing the joint effects for $f_{r}$ increase for high-frequency gain-switching. Detailed studies of the laser dynamics are outside the scope of this paper.

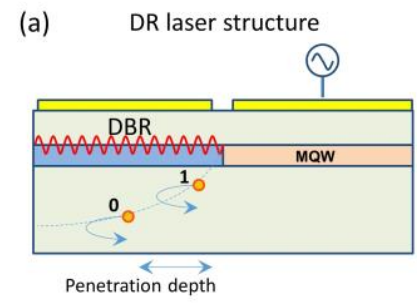

(b) Detuned loading effect

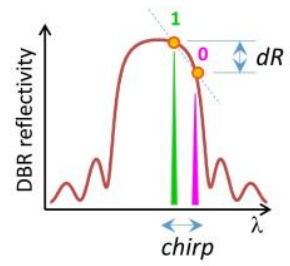

Fig. 1 Detuned-loading effect in distributed reflector (DR) laser [19], where the main lasing mode is located on the long wavelength side of the DBR mirror. 


\section{A. Detuned-loading Effect}

Using DR lasers as example [19], Fig.1a shows the laser structure and the Fig.1b shows the reflectivity of the DBR section at different wavelength. To harness the detuned-loading effect, the wavelength of the main lasing mode generated by the multiple quantum well (MQW) DFB section is positioned at the falling edge of the DBR reflection spectrum.

In a detuned-loaded DBR laser the main lasing mode is located at the falling edge of the long wavelength side of the DBR mirror, as shown in Fig.1b. The increase of the injected current causes a blue shift of the lasing mode towards the reflection peak of the DBR mirror. This reduction of the DBR mirror loss is equivalent to an increase of the gain in the laser section that happens simultaneously with the increase of the carrier density. This simultaneous increase of carrier density and the decrease of cavity loss result in an increased effective differential gain and a reduction of the effective structure chirp factor compared with the material chirp factor [18].

From a laser design point of view, this increase of the effective differential gain is due to the combination of the blue chirp and the slope of DBR mirror. Although the detunedloading effect has been confirmed to improve small-signal modulation BW, the performance for gain-switching, which generates a large transient chirp and thereby changes the detuned loading condition, has not been studied.

\section{B. Photon-photon Resonance Formed by Extended Cavity}

As shown in Fig.1a, for a DR laser with detuned-loading effect, the effective length of the lasing mode is determined by the length of the DFB section and the reflection position in the DBR section. The reflection is weak near the node of DBR mirror, meaning that the light with a longer wavelength (than the main lasing mode) can penetrate deep into the passive DBR section. As a result, the effective cavity length for the side mode is longer than that for the main lasing mode. When the side mode formed by the additional long cavity is located close to the main lasing mode one of the modulation sidebands can be resonantly amplified by the modal resonance. For certain phase conditions the induced carrier density fluctuations can further enhance the fluctuations so the laser starts to self-pulsate. This effect is referred to as PPR and it has been shown to effectively enhance the modulation response near the PPR frequency [19]. The PPR can be generated in DBR lasers, DR lasers or by utilizing a passive feedback section as demonstrated in [22].

\section{Optical Injection Locking}

OIL refers to a technique that external light is injected into the laser cavity such that the slave laser is frequency and phase synchronized to the injected 'master light' [9]. For a given slave laser, both the injection ratio and the detuning frequency affect the output [9]. The injection ratio is usually defined as the power ratio between the master and the slave lasers. The frequency detuning $\left(\Delta \mathrm{f}=\mathrm{f}_{\mathrm{inj}}-\mathrm{f}_{\mathrm{free}}\right)$ is the frequency difference between the injected master light $\left(f_{\text {inj }}\right)$ and the free running slave laser ( $\left.f_{\text {free }}\right)$.

It has been shown that OIL can enhance small-signal modulation BW with a simultaneous reduction of modulation chirp. OIL changes the DML dynamics from the following three aspects: firstly, after OIL, the threshold carrier number is reduced and the photon density is increased [23] and, therefore, the $f_{r}$ is increased. Secondly, OIL creates another PPR that arises from the coupling between the internal DFB mode and the injection light, which correspondingly enhances the modulation response in the high frequency region [24]. Thirdly, it reduces the chirp and ensures that the consecutive pulses are coherent with each other [9].

\section{Discussion of the combined effects}

Although the modulation BW enhancement using the joint effects of the detuned-loading and PPR has been reported [20], the large-signal gain-switching has not been investigated. Furthermore, the possibility to further improve gain-switching repetition rate by utilizing the joint effect of detuned-loading, PPR and OIL has until now not been explored. In gainswitching operation condition, the transient chirp can be up to $100 \mathrm{GHz}$, drastically shifting the wavelength of the main lasing mode with respect to the Bragg reflection peak, changing the dynamic behavior of the detuned-loading effect. Moreover, chirp reduction using OIL adds more complexity to the joint laser dynamics. Therefore, it is our goal to report the experimentally investigates the dynamic behaviours for a more in-depth scientific understanding.

\section{EXPERIMENTS}

\section{A. Cavity-enhanced Laser}

The inset in Fig.2 shows the structure of the cavity-enhanced DFB laser used in this experiment. The so called " $D F B+R$ laser" contains an $80 \mu \mathrm{m}$ length DFB section and an integrated passive waveguide section with a low reflectivity of $\sim 3 \%$ at the front facet. The low front reflection creates a strong filtering effect in the laser cavity, and thus generates both the PPR effect and the detuned-loading effect. As shown in section IV, our cavity-enhanced laser exhibits a small-signal BW of $52.5 \mathrm{GHz}$, whilst the DFB laser fabricated with same design had $24 \mathrm{GHz}$ BW.

The main advantage of the cavity-enhanced laser as compared to the DR laser [20] is the simplicity in operation because it is a single-contact DFB device. The same principle is applicable to longer DFB devices, and therefore suitable for high power and high temperature operation.

\section{B. Experimental Setup}

Fig. 2 shows the experimental setup. The DC bias current and the 50-GHz RF signals were combined with a $55-\mathrm{GHz}$ bias tee and the output was supplied to the laser chip via a $65-\mathrm{GHz}$ RF probe. Both small-signal and large-signal characterizations were performed using this setup. A lightwave component analyzer (LCA, Keysight N5227A) swept the RF frequency from 0.4 to $67 \mathrm{GHz}$ for characterizing the small-signal response. In the large-signal (gain switching) experiments, we generated a $50 \mathrm{GHz}$ sinusoidal signal and boosted the RF power to $30 \mathrm{dBm}$ using a narrow band $(48-52 \mathrm{GHz}) \mathrm{RF}$ amplifier. Due to the RF loss of the bias-tee, RF probe and the carrier substrate, the power of the $50 \mathrm{GHz}$ RF signals that applied on the laser chip was $24 \mathrm{dBm}$, resulting in a peak-to-peak current swing of 
$100 \mathrm{~mA}$.

A 200-kHz-linewidth tunable laser (Santec TSL-550, wavelength accuracy of $\pm 5 \mathrm{pm}$ ) outputting power up to $13 \mathrm{dBm}$ at the telecom O-band was employed as the master laser. The master light was launched into the slave laser via a lens system after passing through a circulator and a polarization controller (PC), which ensured maximum light coupling efficiency to the slave laser cavity. The output of the optical injection locked directly modulated laser (OIL-DML) was emitted from port 3 of the circulator and was split into two branches by a 50:50 splitter. The lower branch was connected to an optical spectrum analyzer (OSA, $0.02 \mathrm{~nm}$ resolution) after attenuation. The upper branch was sent to a Praseodymium-Doped Fiber Amplifier (PDFA). In the small-signal experiment, the optical signals were amplified to $3 \mathrm{dBm}$ for the frequency response measurement. In the gain switching experiment, the PDFA boosted the optical power to $18 \mathrm{dBm}$ for an autocorrelator (Femtochrome FR-103XL) to perform the pulse measurement.

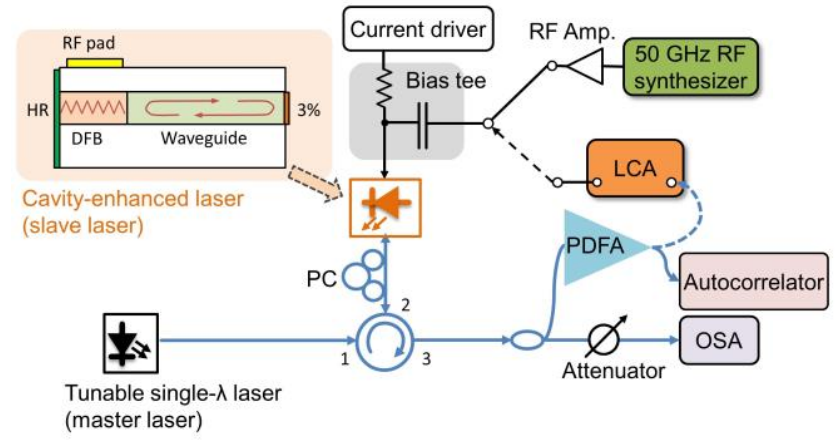

Fig.2 Experimental setup. Inset shows the laser structure. OSA: Optical spectrum analyzer; Amp.: RF amplifier; LCA: Lightwave Component Analyzer; PDFA: Praseodymium-Doped Fiber Amplifier; HR: high reflection; PC: polarization controller; DFB: distributed feedback.

To sufficiently reduce the unwanted chirp for stable and coherent pulse generation, the injection ratio needs to be high enough. However, too much injection power would cause a large locking region that reduces the chirp as well as the detuned-loading effect. In this experiment, we maintained the injection ratio at $-3 \mathrm{~dB}$ to produce stable outputs (i.e. no chaos effect) for enhanced performance in both small-signal and gainswitching experiments. The frequency of the master light was tuned to show the different performance in results in section IV.

\section{RESULTS}

\section{A. Static characterizations}

Fig.3a shows the light-current (LI) curve of the cavity-enhanced laser. The laser has a threshold current of $4.2 \mathrm{~mA}$. The high reflection (HR) coating at the rear facet ensures all the light comes out from the front facet, resulting in a slope efficiency of $0.33 \mathrm{~W} / \mathrm{A}$. Two 'kink points' at 37 and $70 \mathrm{~mA}$ are due to the mode jump from one external mode to another as the frequency of the DFB mode increases with the bias current. Around these conditions, the main lasing mode experiences a strong frequency-dependent cavity loss modulation as the laser is modulated. This detuned-loading effect, described in section IIA, effectively enhances the differential gain and therefore can increase the $f_{r}$. Further increase the bias current beyond $70 \mathrm{~mA}$ leads to a decrease of the output power due to the thermal effects. Fig.3b shows the optical spectrum when biasing at $65 \mathrm{~mA}$, where the PPR mode is $34 \mathrm{GHz}$ lower than the lasing frequency, leading to enhancement of the high frequency components.
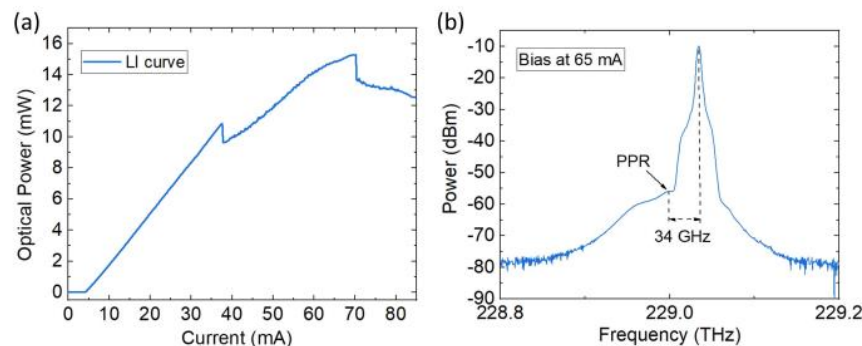

Fig.3 (a) Light-current (LI) curve of the cavity-enhanced laser; (b) Optical spectrum when biasing at $65 \mathrm{~mA}$ (resolution of $0.02 \mathrm{~nm}$ ).

\section{B. Small-signal responses}

Fig. 4 shows the small-signal frequency response of the cavityenhanced laser without OIL. As the bias current increases from $55 \mathrm{~mA}$ (red line) to $65 \mathrm{~mA}$ (black short dashed line), the oscillation frequency was increased from $25 \mathrm{GHz}$ to about $34 \mathrm{GHz}$, leading to an increase of the modulation BW from $39 \mathrm{GHz}$ to $52 \mathrm{GHz}$. Further increasing the bias current to $69 \mathrm{~mA}$ (blue dashed line) resulted in strong oscillation (selfpulsation) in the frequency range of $40-45 \mathrm{GHz}$, making it unsuitable for data modulation. The dip seen at $44 \mathrm{GHz}$ is due to a parasitic RF resonance of the chip-on-carrier. Noting that, the strong oscillation when biasing at $69 \mathrm{~mA}$ is not suitable for data modulation because the strong ringing at the $f_{r}$. The nonflat frequency response also causes signal distortion and intersymbol interference for broadband modulations. A further increase of the bias current to $71 \mathrm{~mA}$ resulted in a mode jump and the PPR effect disappeared, causing a reduction of BW to $27 \mathrm{GHz}$ (green solid line).

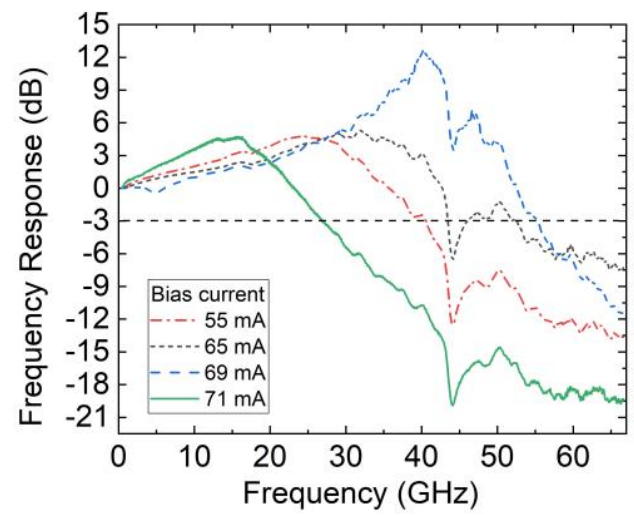

Fig. 4 Frequency responses of the cavity-enhanced laser at different bias current. Red Dash dot line: $55 \mathrm{~mA}$; short dashed line: $65 \mathrm{~mA}$; dashed line: $69 \mathrm{~mA}$; Green solid line: $71 \mathrm{~mA}$. The responses at low frequency $(0.4 \mathrm{GHz})$ are normalized to $0 \mathrm{~dB}$ to compare the small-signal BW. The dip at $44 \mathrm{GHz}$ is caused by a parasitic RF resonance of the chip-on-carrier.

Fig.5a shows the optical spectra of the cavity-enhanced laser with OIL for different detuning frequencies. The slave laser was biased at $65 \mathrm{~mA}$, outputting $14 \mathrm{~mW}$ at $229.03 \mathrm{THz}$ $(1308.96 \mathrm{~nm})$. To study the impact of OIL on the small-signal responses, the center frequencies of the captured optical spectra 
have been shifted to $0 \mathrm{GHz}$. The green, red, and blue curves show the spectra of the OIL-DML with frequency detuning values of +3 (green), -6 (red), and $-15 \mathrm{GHz}$ (blue), respectively. Their corresponding frequency response measurements $(0.4-$ $67 \mathrm{GHz}$ ) are shown as the same color in Fig.5b. The optical spectra and the frequency response of the solitary laser without OIL are shown in both Fig.5a and Fig.5b as the black dashed lines. The inset in Fig.5a zooms in the optical spectra in the range of -25 to $-65 \mathrm{GHz}$ to show the enhancement of the intensity of the optical side modes.
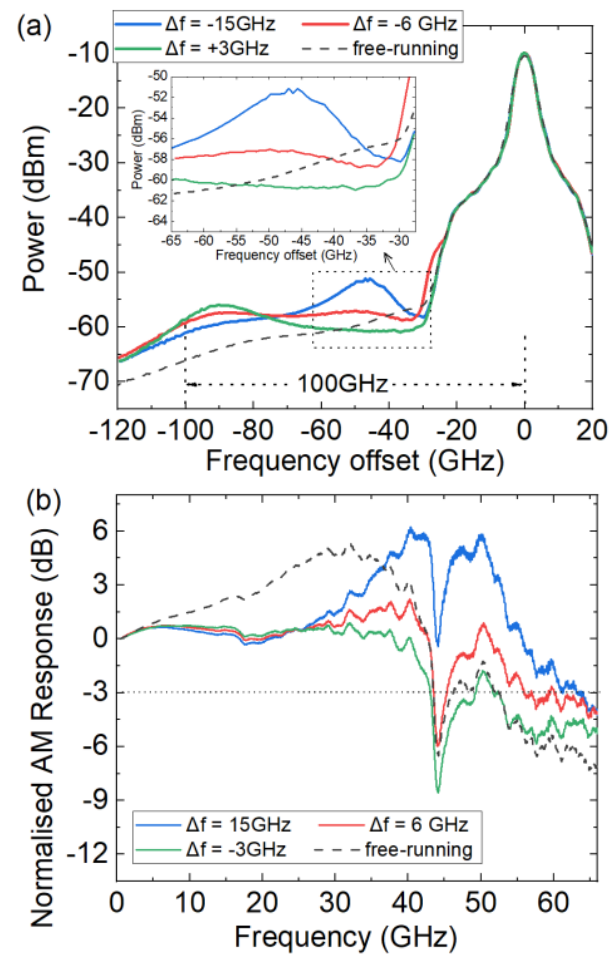

Fig. 5 (a) Optical spectra of the slave laser output with different OIL detuning frequencies; (b) Frequency responses (normalized at $0.4 \mathrm{GHz}$ ) at different OIL-DML detuning frequencies. The solitary results at $65 \mathrm{~mA}$ are plotted as the black dashed in as reference. The dip at $44 \mathrm{GHz}$ is caused by a parasitic RF resonance of the chip-on-carrier

At the frequency detuning of $-15 \mathrm{GHz}$, the OIL induced resonance located at around $45 \mathrm{GHz}$ (blue line in Fig.5a), leading to a significantly enhanced frequency response between 40 and $55 \mathrm{GHz}$, as shown by the blue curves in Fig.5b. When reducing the frequency detuning to $-6 \mathrm{GHz}$ (red curves), the resonance frequency and the damping changed accordingly [24]. The joint effects of both detuned-loading, PPR and OIL led to a flat optical side mode with less than $2 \mathrm{~dB}$ power fluctuation from 35 to $100 \mathrm{GHz}$, and the corresponding frequency response also becomes flat within the measured range, as shown by the red curve in Fig.5a. Further shifting the frequency detuning to $+3 \mathrm{GHz}$ led to a strong OIL resonance at $90 \mathrm{GHz}$, shown as the green curves in Fig.5a. A corresponding reduction of frequency response between 35 and $67 \mathrm{GHz}$ is shown in the frequency response in Fig.5b. At the OIL detuning frequencies of both -6 and $+3 \mathrm{GHz}$, an optical side band up to $100 \mathrm{GHz}$ was observed from the optical spectra.

\section{Gain switching}

Fig. 6 shows the LI characteristics of the cavity-enhanced laser under gain switching as red dashed line. The LI curve under DC drive shown in Fig.3a is plotted for comparison. To avoid the damage on the laser modulated under high RF power without DC bias, we did not measure the threshold current under gain switching. Under gain switching, only one kink around $55 \mathrm{~mA}$ was observed due to the higher local Joule heating in the DFB section under large RF modulation. Thus, in the gain-switching experiment, the DC current was lowered to $52-54 \mathrm{~mA}$, where the detuned-loading effect occurs. The output optical power was around $11 \mathrm{~mW}$.

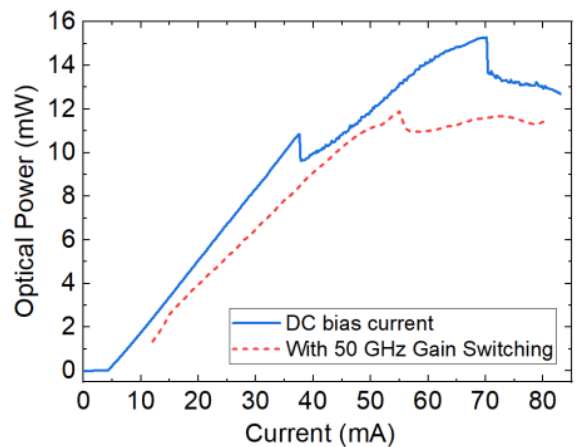

Fig. 6 Light-current measurement (LI curve) of the solitary laser under DC current drive (blue solid line) and 50-GHz gain switching (red dashed line).

Fig. 7 shows the optical spectrum of the gain switched DML without OIL. The DML was biased at $52 \mathrm{~mA}$ for obtaining the shortest pulse width. Although we obtained clear 50-GHzspacing tones, some residual chirp still existed, as shown by the frequency components between the tones.

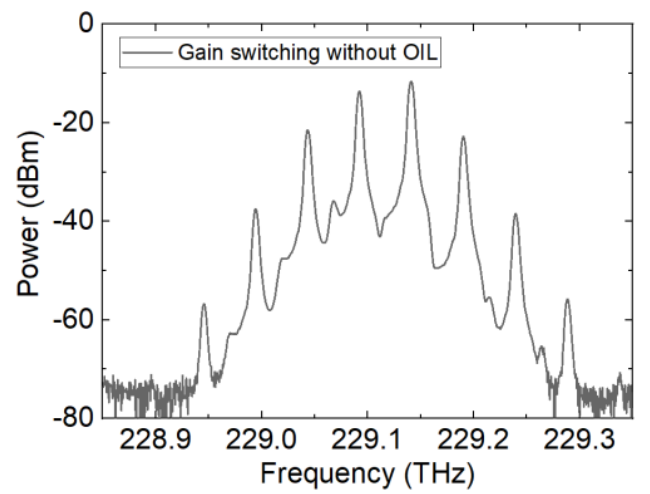

Fig. 7 Optical spectrum of the gain switched DML without OIL. The DML was biased at $52 \mathrm{~mA}$.
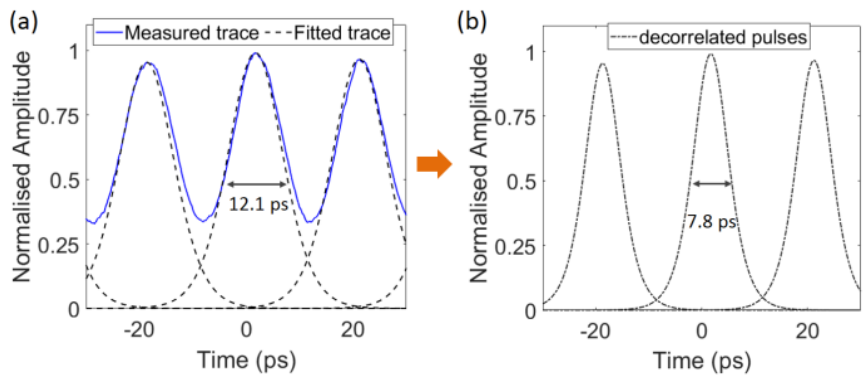

Fig. 8 Gain switching using the cavity-enhanced laser without OIL. (a) Measured autocorrelator results (blue curve) and the fitted pulse train (dashed curves) after removing the overlaps; (b) decorrelated pulses assuming $\operatorname{Sech}^{2}$ pulse shape.

The blue solid curve in Fig. 8a shows the measurements from 
the autocorrelator. Due to the high repetition rate, the pedals of consecutive pulses are overlapped. Assuming $\operatorname{sech}^{2}$ pulse shape, we decompose the pulses and plot them as black dashed lines in Fig. 8a, resulting in a full width half maximum (FWHM) value of $12.1 \mathrm{ps}$. After decorrelation, the pulse width is 7.8 ps, as shown in Fig.7b. Note that Fig. 8 auto-correlation shows slightly weaker pulses on both sides of center pulse, indicating that weak period-doubling occurred in the gainswitching. This can also be confirmed from the optical spectrum where a weak frequency tone (about $20 \mathrm{~dB}$ lower) can be observed at the center of two adjacent 50-GHz-spaced tones. This period doubling occurs when the $f_{r}$ is lower than the gainswitching frequency. The optical spectrum of the chirped signal has a FWHM of $93 \mathrm{GHz}$ and the corresponding time-bandwidth product was 0.71 .

Fig. 9 shows the optical spectrum of the gain switched DML with OIL. The OIL suppressed the chirp and resulted in resolved 50-GHz spacing tones and a SMSR of $23 \mathrm{~dB}$. The power variation of the three center lines was only $1 \mathrm{~dB}$, making it a promising light source for flat comb generation with the use of expansion techniques [10]. The time domain performance measured using the autocorrelator is shown in Fig.10a. The FWHM value with was $9.5 \mathrm{ps}$ before decorrelation. Assuming $\mathrm{sech}^{2}$ shape, the calculated pulse widths is $6.2 \mathrm{ps}$ (Fig.10b). Compared to previous gain-switched demonstration at $20 \mathrm{GHz}$ that showed a pulse width of $12.3 \mathrm{ps}$ with an $f_{r}$ of $25 \mathrm{GHz}$ [25], we achieved a reduction of pulse width by a factor of nearly 2 . The relaxation oscillation frequency is improved by about the same factor. The spectrum of the chirped optical signal has a FWHM of about $130 \mathrm{GHz}$, corresponding to a time-BW product of 0.81 , Assuming linear chirp, the pulse width can be compressed to $2.4 \mathrm{ps}$.

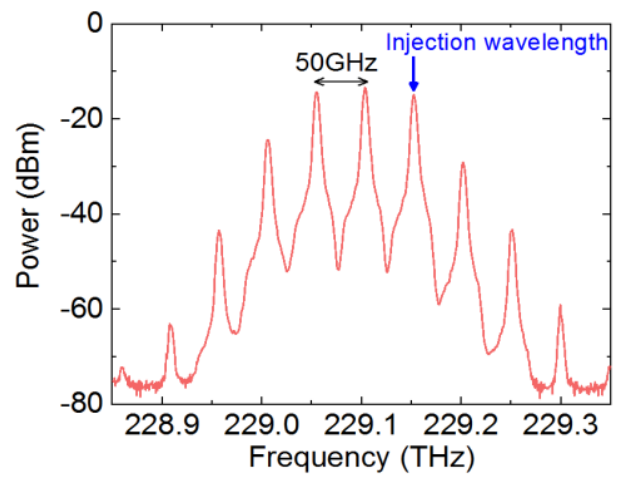

Fig. 9 Optical spectra of the gain-switched DML with OIL at $299.15 \mathrm{THz}$.

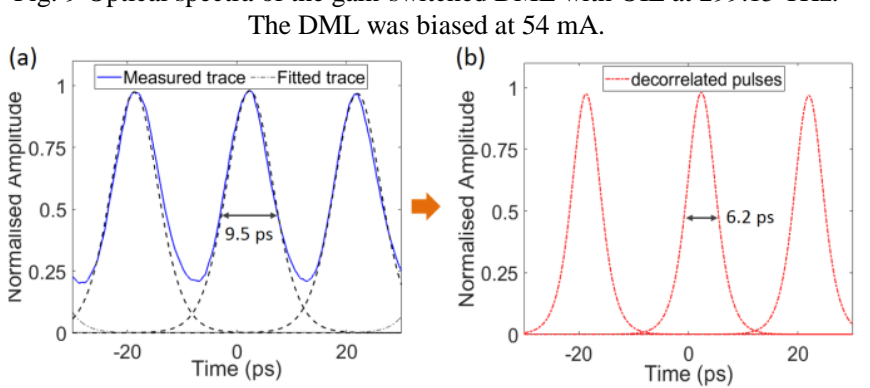

Fig.10 Gain switching using the cavity-enhanced laser with OIL. (a) Measured autocorrelator results (blue curve) and the fitted pulse train (dashed curves) after removing the overlaps; (b) decorrelated pulses assuming $\operatorname{Sech}^{2}$ pulse shape.

\section{CONCLUSION}

We study the combined effects of detuned-loading, PPR and OIL for gain-switching DFB laser, achieving pulse generation at a record high repetition rate of $50 \mathrm{GHz}$ and an uncompressed pulse width of $6.2 \mathrm{ps}$. The results confirm that the detunedloading, PPR and OIL can improve the relaxation oscillation frequency in large-signal modulation. We observed from the optical spectra that the OIL creates a second PPR that results in a flat optical sideband with less than $2 \mathrm{~dB}$ power fluctuation up to $100 \mathrm{GHz}$, which could be potentially explored for high-speed direct laser modulation. The results confirm that the cavityenhanced OIL-DML is suitable as an optical pulse source (or frequency comb) for high speed data transmission.

\section{ACKNOWLEDGMENT}

The authors thank Femtochrome for loan of the autocorrelator.

\section{REFERENCES}

[1] Y. Matsui, "Directly Modulated Laser Technology: Past, Present, and Future," Datacenter Connectivity Technologies: Principles and Practice, Gistrup, Demark: River Publisher, 2018, ch.3, pp. 87.

[2] K. Sato, S. Kuwahara, and Y. Miyamoto, "Chirp Characteristics of 40$\mathrm{Gb} / \mathrm{s}$ Directly Modulated Distributed-Feedback Laser Diodes," IEEE/OSA J. Light. Technol., vol. 23, no. 11, pp. 3790-3797, 2005.

[3] K. Y. Lau, "Gain switching of semiconductor injection lasers," Appl. Phys. Lett., vol. 52, no.4, pp.257-259, 1988.

[4] R. A. Linke, "Modulation Induced Transient Chirping in Single Frequency Lasers," IEEE J. Quantum Electron, vol. QE-21, No.6, 593597, 1985.

[5] Y. Matsui, M. D. Pelusi, and A. Suzuki, "Generation of 20-fs Optical Pulses from a Gain-Switched Laser Diode by a Four-Stage Soliton Compression Technique," IEEE Photon. Technol. Lett., vol. 11, no. 10, pp. 1217-1219, 1999.

[6] S. Kawanishi, "Ultrahigh-speed optical time-division-multiplexed transmission technology based on optical signal processing," IEEE/OSA J. Light. Technol., vol. 34, no. 11, pp. 2064 - 2079, 1998.

[7] L. C. Comandar, M. Lucamarini, B. Fröhlich, et al., "Quantum key distribution without detector vulnerabilities using optically seeded lasers," Nature Photonics, vol. 10, pp. 312-315, 2016.

[8] C. Deakin and Z. Liu, "Dual frequency comb assisted analog-to-digital conversion," vol. 45, no. 1, pp. 173-176, 2020.

[9] Z. Liu and R. Slavík, "Optical Injection Locking: from principle to applications" J. Lightw. Technol., vol.30, no.1, pp. 43-59, 2020.

[10] P. M. Anandarajah, R. Maher, Y.Q. Xu et al., "Generation of coherent multicarrier signals by gain switching of discrete mode lasers, " IEEE Photonics J., vol. 3, no.1, pp 112-122, 2011.

[11] R. Zhou, S. Latkowski, J. O'Carroll et al., "40nm wavelength tunable gain-switched optical comb source," Opt. Express, vol. 19, no. 26, pp B415-B420, 2011.

[12] P. M. Anandarajah, R. Zhou, R. Maher et al., "Flexible Optical Comb Source for Super Channel Systems," Optical Fiber Communication Conference (OFC), paper OTh3I.8, Anaheim, 2013.

[13] J. Yu, X. Zhou, "Ultra-High-Capacity DWDM transmission system for 100G and beyond," IEEE Commun. Mag., vol. 48, no.4, pp. S56-S64, 2010.

[14] P. Marin-Palomo, J.N. Kemal, M. Karpov et al., "Microresonator-based solitons for massively parallel coherent optical communications," Nature, vol. 546, pp. 274-279, 2017.

[15] Y. Matsui, S. Kutsuzawa, S. Arahira et al., "Generation of wavelength tunable gain-switched pulses from FP MQW lasers with external injection seeding," IEEE Photon. Technol. Lett., vol. 9, no.8, pp. 1087-1089, 1997.

[16] J. Sacher, D. Baums, P. Panknin et al., "Intensity instabilities of semiconductor lasers under current modulation, external light injection, and delayed feedback," Phys. Rev. A, vol. 45, no. 3, pp. 1893-1905, Feb. 1992.

[17] J. Kreissl, V. Vercesi, U. Troppenz, T. Gaertner, W. Wenisch, and M. Schell, "Up to 40-Gb/s Directly Modulated Laser Operating at Low Driving Current: Buried-Heterostructure Passive Feedback Laser (BH- 
PFL),” IEEE Photon. Technol. Lett., vol. 24, no.5, pp. 362-364, Mar. 2011.

[18] M. Chaciński and R. Schatz, "Impact of losses in the Bragg section on the dynamics of detuned loaded DBR lasers," IEEE J. Quantum Electron, vol. 46, no. 9, pp. 1360-1367, Sep. 2010.

[19] Y. Matsui, R. Schatz, T. Pham, W. A. Ling, G. Carey, H. M. Daghighian, D. Adams, T. Sudo, and C. Roxlo, "55 GHz BW Distributed Reflector Laser," IEEE/OSA J. Light. Technol., vol. 35, no. 3, pp 397-403, 2017.

[20] D. Che, Y. Matsui, R. Shatz et al., "Direct Modulation of a 54-GHz Distributed Bragg Reflector Laser with 100-GBaud PAM-4 and 80GBaud PAM8," Optical Fiber Communication Conference (OFC), paper Th3C.1, San Diego, 2020.

[21] S. Yamaoka1, N. Diamantopoulos, H. Nishi et al., "239.3-Gbit/s Net Rate PAM-4 Transmission Using Directly Modulated Membrane Lasers on High-thermal-conductivity SiC," European Conference of Optical Communications (ECOC), paper PD.2.1, 2019.

[22] M. Radziunas, A. Glitzky, U. Bandelow, M. Wolfrum, U. Troppenz, J. Kreissl, and W. Rehbein, "Improving the Modulation Bandwidth in Semiconductor Lasers by Passive Feedback," J. Sel. Top. Quantum Electron., vol. 13, no. 1, pp 136-142, 2007.

[23] A. Murakami, K. Kawashima, and K. Atsuki, "Cavity resonance shift and bandwdith enhancement in semiconductor lasers with strong light injection," IEEE J. Quantum Electron., vol. 39, no. 10, pp 1196-1204, 2003.

[24] E. K. Lau, L. J. Wong, and M. C. Wu, "Enhanced Modulation Characteristics of Optical Injection-Locked Lasers: A Tutorial," J. Sel. Top. Quantum Electron, vol. 15, no. 3, pp. 618-633, 2009.

[25] Y. Matsui, S. Kutsuzawa, S. Arahira, Y. Ogawa, and A. Suzuki, "Bifurcation in 20-GHz Gain-Switched 1.55- m MQW Lasers and Its Control by CW Injection Seeding," IEEE J. Quantum Electron, vol.34, no.7, pp 1213-1223, 1998. 